\title{
Research on Micro Energy Collection for Building Energy Saving Prediction
}

\author{
Hong Zhang ${ }^{1, \text { a }}$ \\ ${ }^{1}$ Shandong Institute of Commerce and Technology, Jinan 250103, China \\ a email: zhanghongsd78@163.com
}

\section{Keywords: Energy Conservation; Building; Air Conditioning; Collection Of Micro Energy}

\begin{abstract}
The diversity and complexity of large public buildings, our country's local climate, economy, life habits variation result in a lack of basic data to support energy-saving operation standard, energy saving technology for the reconstruction of the applicability and the lack of practical test, which leads to a phenomenon. Operating standards is still derived from the design standards and a small number of measured data, energy-saving technological transformation in a project or place successfully is popularized in the whole country, the engineering application and theory of scientific research confusion is not clear, management of government departments, public building owners is at a loss.
\end{abstract}

\section{Building Energy Efficiency Mode}

In order to determine whether the research in this chapter summarizes the key energy-saving buildings on the common defects, this study randomly selected a key buildings before carrying out the basis of auditing and testing in accordance with the results of research in this chapter which is determined the transformation plan and the efficacy of each scheme through actual audit and inspection.

To realize the energy saving operation of the air conditioning system, it must be up from an overall analysis for evaluation of the air conditioning system operation condition, but the complexity of the air-conditioning system itself makes the analysis and evaluation of it is very difficult to implement, it is necessary to form a system of analysis and evaluation methods. Air treatment systems to maintain air conditioning room air in a specific state to meet the requirements of air conditioning room temperature, humidity and air quality in the use of air conditioning room. Air treatment system and air conditioning room are directly linked. The fluid transport system is a pipeline transportation system which uses the circulation flow of the heat medium to transfer the cold / heat generation to the air treatment equipment. Cold and heat source room is the preparation of air conditioning system to provide cold heat, which is the entire air-conditioning system of the cold heat supply side.

outer environment

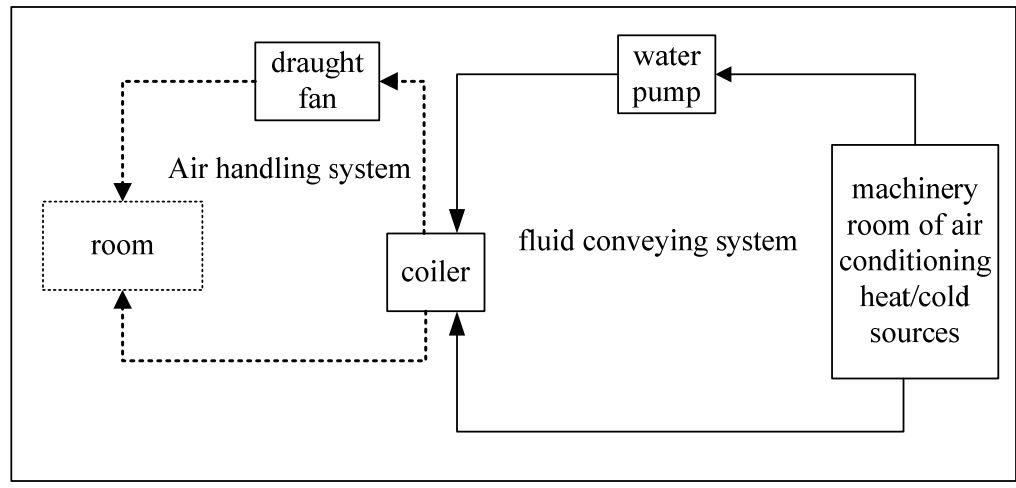

Fig.1. Air conditioning system diagram 


\section{Air Conditioning to Fine Tune The Collection of Energy Saving}

The goal of our research is to ensure that the air conditioning system as a whole is in a state of energy conservation, the overall energy of the air conditioning can be reduced as far as possible. Through the analysis based on the detection of load simulation of room air conditioning energy-saving diagnosis and air conditioning equipment, we believe that the air-conditioned room in the energy-saving running state, air conditioning equipment can be in normal operation. Based on such a premise and foundation, the overall air-conditioning energy saving diagnosis method can have greater choice of space, this is our design the main idea of the whole air conditioning system energy efficiency diagnosis method. Air-conditioning in the overall energy saving diagnosis method can choose the space after expanding, how should choose what kind of method, how to come up with this method must also be from the aspects of the mode of operation of the air conditioning system, especially in the room air-conditioning energy-saving operation. The normal operation of the air conditioning equipment is under the premise of air conditioning system operation mode and clear air conditioning system energy, by which factors influence only in this way that can ensure the overall energy saving operation of the air conditioning system in measure method. Here we will from the operation of the air conditioning system, analysis is given by specific energy-saving diagnosis.

Defining "Energy Consumption ECR" (Rate) to evaluate the energy efficiency of the transformation of economic.ECR is defined as: under the conditions of the transformation of the ideal energy consumption and the actual energy consumption ratio, that is, ECR

$$
E C R=\frac{W}{W_{C}}
$$

In the formula, $\mathrm{W}$ is for air conditioning system energy consumption; no index that the transformation conditions, the index $\mathrm{C}$ for the actual conditions. Among them:

$$
\left\{\begin{array}{l}
W=W_{1}+W_{2}+W_{3}+W_{4}+W_{5} \\
W_{C}=W_{C 1}+W_{C 2}+W_{C 3}+W_{C 4}+W_{C 5}
\end{array}\right.
$$

In the formula, $1,2,3,4,5$ respectively represent the end of the fan, refrigeration water cycle pump, chiller, cooling water cycle pump, cooling tower fan (or air-cooled condenser fan).

Air conditioning process itself is a cold heat transfer process, which contains a number of hot media flow cycle, heat transfer in each of the heat media cycle that is reflected in two related variables: flow and temperature difference. The general air conditioning system can be abstracted as the form given in Figure 2.

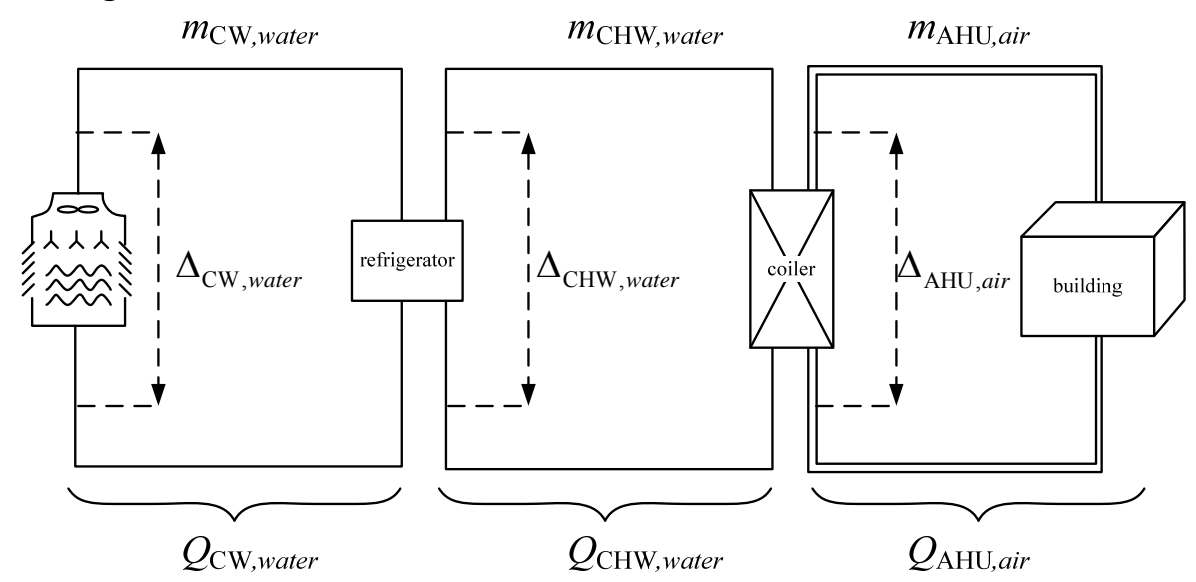

Fig.2. Schematic diagram of the general air conditioning refrigeration system

$\mathrm{G}(\mathrm{V}, \mathrm{E})$ represents a wireless sensor network, where $\mathrm{V}$ is composed of nodes in the network of non empty set, e is the set of radio links in a network (also known as the, and G (V, e)which is non-connected with weighted graph. In wireless sensor networks, if the node $\mathrm{j}$ in the communication radius is of node $i$, the node $j$ is the neighbor node of node $I$. At this point, the node 
I is also a neighbor node of the node j. Link (I, J) indicates the node $i$ to the node $j$ link. N (I) represents a collection of all the neighbor nodes of the node I. When it meets the demand of air conditioned room, the air conditioning system provides the cold heat of the air conditioning system. We think that the running state of the air conditioning system is fixed, that is to say, the air-conditioning system has a fixed response mode. Based on this hypothesis, through the analysis of set point set different values when air conditioning system supplies the same cold heat air conditioning system total energy changes, you can determine the pros and cons of the set value and puts forward based on the set value of fine-tuning of the whole air conditioning system energy saving diagnosis method.

As a result of the implementation of the "air-conditioning supplies, the same cold heat time call energy comparison links can also be analyzed by data distribution method and the graphical analysis method is more intuitive, which is easy to understand and operating the diagram method of observation. In the following analysis, the feasibility of the method of air-conditioning energy saving diagnosis is based on the setting value adjustment which is verified by the graphic observation method. Wireless sensor network is a low cost, battery powered wireless network, it has the function of data sensing and wireless communication. Power consumption has been a key issue in wireless sensor networks, because it directly affects the survival time of the network. Due to the limited energy, the nodes rely on short distance communication and the data is converged to the Sink node through the multi hop network. Therefore, in wireless sensor networks, data collection algorithm is a challenging problem. It is mainly for the data source nodes (such as sensor nodes) and data Sink nodes (such as the base station) to provide energy consumption and reliable path.

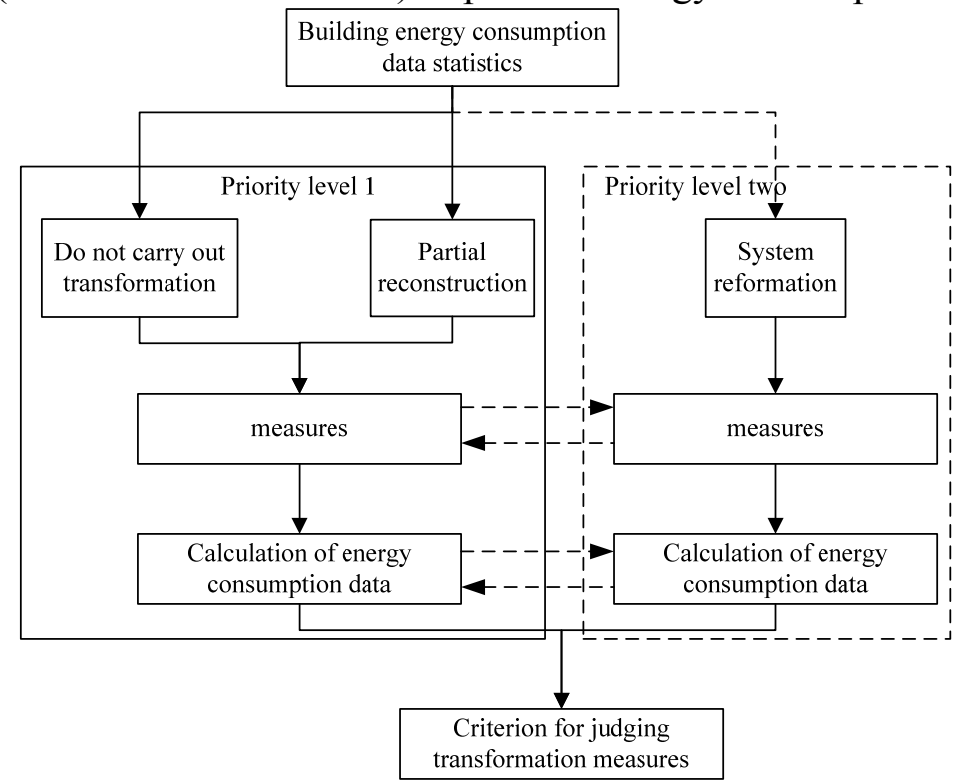

Fig.3. the basis of energy saving

\section{Experiment}

Total construction area is about 33000 square meters, with air conditioning area of 23000 square meters. the 8 floor (underground garage, kitchen, room). Main building is for office use, catering, conference purposes. The building's air conditioning system in centrifugal chillers as cold source; water delivery system is for a pump constant flow chilled water pump system; wind conveying system for fan coil unit plus fresh air combined form, air conditioning engineering equipment configuration (see Appendix). According to the cooler 2007 annual operation records, in 2007 a total of stand-alone operation hours is about 2165 hours, the transition season running a total of 120 hours, accounting for only year-round operation of about $5.5 \%$, with air conditioning system operation 1920 hours. In a cooling season run, in August seven, part of high temperature weather need to open two Taiwan refrigerator. Single chiller load rate is only $50 \%$ of the time which is greater than $70 \%$, the load rate is low. Select the July test results of the cooling water inlet 
temperature in the $26.50 \mathrm{C} \sim 310 \mathrm{C}$, the water temperature is basically maintained at $70 \mathrm{C} \sim 8.50 \mathrm{C}$. The measurement results show that the load rate varies from $81 \%$ to $107 \%$, but the change range of COP is between 2.6 and 5.7. The monitoring results can be found that the temperature of the cooling water is kept constant and the load rate of the main engine is not obvious. Retrofit scheme: such as the use of building wind balance reconstruction scheme, shortening the time window to fan coil processing load of fresh air, in the air humidity is improving measures of indoor temperature, especially for that the building fresh air system is not controlled, constant flow, constant air volume, design of the pipeline is caused by unreasonable amount of fresh air which are serious and can effectively reduce the load of fresh air.

\section{Conclusion}

Through the verification of the project, eight measures can be implemented, accounting for the original scheduled $60 \%$ of the total reservation scheme. Although part of the program's implementation is at the same time, implementation measures should be less than $60 \%$, but the conclusion is enough to illustrate that this chapter summarizes the key energy-saving which has universality as its focus on building energy audit is feasible.

\section{References}

[1] Guanqun Bao, Liang Mi, Yishuang Geng, Kaveh Pahlavan, A computer vision based speed estimation technique for localiz ing the wireless capsule endoscope inside small intestine, 36th Annual International Conference of the IEEE Engineering in Medicine and Biology Society (EMBC), Aug. 2014

[2] Xinchao Song, Yishuang Geng, Distributed community detection optimization algorithm for complex networks, Journal of Networks, 9(10), 2758-2765, Jan. 2014.

[3] Jiang D, Ying X, Han Y, et al. Collaborative multi-hop routing in cognitive wireless networks[J]. Wireless Personal Communications, 2015: 1-23.

[4] Jinyu $\mathrm{Hu}$ and Zhiwei Gao. Modules identification in gene positive networks of hepatocellular carcinoma using Pearson agglomerative method and Pearson cohesion coupling modularity[J]. Journal of Applied Mathematics, 2012 (2012). 